\title{
NEW BIOTECHNOLOGICAL TREATMENTS FOR LIPID DISORDERS
}

\author{
Natalia Valencia-Enciso ${ }^{1}$ and Carlos O. MendiviL ${ }^{1,2}$ \\ ${ }^{1}$ Department of Medicine, Universidad de los Andes School of Medicine; ${ }^{2}$ Department of Internal Medicine, \\ Endocrinology Section, Fundación Santa Fe de Bogotá, Bogotá, Colombia
}

\begin{abstract}
Disorders of lipid and lipoprotein metabolism play a central role in the pathogenesis of atherosclerotic cardiovascular diseases (CVDs). Despite the widespread use of efficacious lipid-modifying therapies, the residual risk of CVD remains unacceptably high. The purpose of this manuscript is to review the application of new technologies in the treatment of lipid disorders. New therapies work mostly at the gene expression level and are, therefore, different from traditional small-molecule drugs that work mainly by inhibiting already synthesized proteins. We will briefly lay out the function of the gene products targeted by the new agents. Then, we will organize our review of new biotechnological treatments by the molecular approach, namely: monoclonal antibodies, antisense oligonucleotides, small-interfering RNAs, and Clustered Regularly Interspaced Short Palindromic Repeats (CRISPR)/CRISPR-associated 9 (Cas9)-based genome editing. The paper concludes with the description of the current clinical studies and the perspectives for the use of these agents. (REV INVEST CLIN. 2018;70:244-54)
\end{abstract}

Key words: Monoclonal antibodies. Antisense oligonucleotides. Small-interfering RNAs.

\section{INTRODUCTION}

Disorders of lipid and lipoprotein metabolism play a central role in the pathogenesis of atherosclerotic cardiovascular diseases (CVDs). Despite the widespread use of efficacious lipid-modifying therapies, the residual risk of CVD remains unacceptably high ${ }^{1}$. Besides suboptimal control of non-lipid risk factors, our ability to reduce CVD is also constrained by intolerance or poor adherence to statin therapy ${ }^{2}$, limited availability and access to therapies for severe, genetic dyslipidemias ${ }^{3}$, and inconsistent evidence about

Corresponding author:

*Carlos O. Mendivil

Universidad de los Andes, School of Medicine

Carrera 7 No 116-05, Of. 413

Bogotá, Colombia

E-mail: cmendivi@uniandes.edu.co the benefits of impacting the high-density lipoprotein (HDL)/triglycerides (TG) axis ${ }^{4}$.

New and promising pharmacological agents employ tools from biotechnology and molecular biology to have a positive impact on lipid metabolism. These new treatments for lipid disorders work mostly at the gene expression level and are, therefore, different from traditional small-molecule drugs that mainly act by inhibiting already synthesized proteins. We will briefly lay out the function of the gene products targeted by the new agents. Then, we will organize our

Received for publication: 02-05-2018 Approved for publication: 13-06-2018 doi: $10.24875 / R I C .18002577$ 
review of new biotechnological treatments by their molecular approach, namely: monoclonal antibodies (mAb), antisense oligonucleotides (ASO), small-interfering RNAs, and Clustered Regularly Interspaced Short Palindromic Repeats (CRISPR)/CRISPR-associated 9 (Cas9)-based genome editing.

\section{MOLECULAR TARGETS}

\section{APOB}

The $A P O B$ gene encodes apolipoprotein $B(a p o B$ ), the structural apo of chylomicrons (although in the chylomicron-specific version only the initial $48 \%$ of the apoB mRNA is translated), very low-density lipoproteins (VLDLs), intermediate-density lipoproteins (IDLs), and LDL. Agents aimed at limiting the hepatic production of apoB can reduce the concentrations of all these atherogenic lipoproteins.

\section{PCSK9}

The PCSK9 gene encodes proprotein convertase subtilisin/kexin type 9 , an enzyme whose physiological role is to impede the recycling of $L D L$ receptors (LDLr) from endocytic vesicles to the plasma membrane of hepatocytes. Thus, PCSK9 effectively reduces the number of LDLr present on the membrane and increases plasma LDL concentrations. In 2003, a new type of autosomal dominant familial hypercholesterolemia was identified ${ }^{5}$. The mutated gene mapped to the short arm of chromosome 1 and was later recognized as the gene for PCSK $9^{6}$. It was soon discovered that gain-of-function mutations in PCSK9 result in a large decrease in the hepatic expression of LDLr through a post-translational mechanism ${ }^{7}$. Subsequently, a genetic study in African-Americans reported an association between two new nonsense PCSK 9 mutations and substantial reductions in plasma LDL cholesterol (LDL-C) and a reduced occurrence of $\mathrm{CVD}^{8}$. Other nonsense or missense mutations in PCSK9 were discovered, all of which were effectively associated with large reductions in LDL-C and reduced $\mathrm{CVD}^{9,10}$. The mechanism by which PCSK9 alters the expression of LDLr was elucidated in 2007: the catalytic domain of PCSK9 binds the LDLr extracellularly. Once the PCSK9-LDL-LDLr complex is endocytosed, PCSK9 prevents the dissociation of LDL and LDLr, channeling the LDL $r$ to lysosomes for its degradation ${ }^{11}$.

\section{ANGPTL3}

The ANGPTL3 gene encodes angiopoietin-like 3, a protein secreted mostly by the liver that acts as an inhibitor to lipoprotein-lipase (LPL-1) and endothelial lipase $^{12}$. ANGPTL3 inhibition or prevention of its production has the potential to reduce plasma TG. By inhibiting LPL-1, ANGPTL3 prevents the hydrolysis and clearance of circulating TG from chylomicrons and VLDL ${ }^{13}$. The interaction of ANGPTL3 with LPL-1 is dependent on the formation of a complex with ANGPTL8, a sister protein ubiquitously expressed in various cell types ${ }^{14}$. Humans with loss-of-function mutations in ANGPTL3 present clinically with familial combined hypolipidemia. This phenotype includes reduced plasma total cholesterol, TG, and LDL-C, reduced coronary plaque burden, and a lower risk of CVD ${ }^{15}$. The lifetime reduction in CVD risk among patients with lossof-function ANGPTL3 variants has been estimated at $41 \%{ }^{16}$. Subjects homozygous for loss-of-function mutations in ANGPTL3 also exhibit lower glycemia, insulinemia, and less insulin resistance ${ }^{17}$.

\section{APOC3}

The APOC3 gene encodes apoC-III, a small interchangeable apo that interferes with the removal of VLDL, IDL, and LDL by hepatic receptors. Antagonism or blockade of apoC-III increase liver extraction of plasma lipoproteins and reduce plasma cholesterol and TG. ApoC-III antagonizes binding of apoE to the LDLr, promoting accumulation of atherogenic lipoproteins in circulation ${ }^{18,19}$. Further, the atherogenicity of LDL can be almost totally accounted for by LDL that contains apoC-III' ${ }^{20}$, and $\mathrm{HDL}$ that contains apoC-III has a positive, not negative association with CVD risk $^{21}$. Mutations in the APOC 3 gene are associated with lower plasma TG levels, lower CVD risk, and greater longevity 22 .

\section{LPA}

The LPA gene encodes apo(a), an inactive serine proteinase with procoagulant activity, through inhibition of tissue-type plasminogen activator. In circulation, apo(a) is present as part of lipoprotein (a) (Lp[a]), a macromolecular structure with similarity to LDL that includes a lipid cargo and an apo(a) molecule covalently bound to apoB. Each molecule of apo(a) has a genetically determined number of kringle-type 
domains (usually 2-43), which impact its physical properties ${ }^{23}$. Genetic and epidemiological evidence suggests an independent, causal role for $\operatorname{Lp}(a)$ as a promoter of atherosclerosis ${ }^{24,25}$, but the molecular mechanism behind such association is not entirely clear.

\section{Monoclonal Antibodies (mAb)}

The development of hybridoma technology in $1975^{26}$ has given way to the mass production of $\mathrm{mAb}$ against virtually any given protein of interest. A notable feature of mAbs is their long half-life and ability to bind their target with great specificity.

\section{mAb against PCSK9}

Binding of the mAb to the active site of PCSK9 or a nearby domain results in increased recycling of LDLr to the cell surface, faster hepatic uptake of LDL, and reduced plasma $\mathrm{LDL}-\mathrm{C}^{27}$.

\section{Evolocumab}

Evolocumab is a fully human mAb against PCSK9. Evolocumab consists of two heavy chains and two light chains of the lambda subclass; evolocumab does not contain an Fc region.

\section{EFFICACY OF EVOLOCUMAB}

Patients with heterozygous familial hypercholesterolemia ( $\mathrm{HeFH}$ )

The RUTHERFORD-2 trial evaluated the efficacy and safety of evolocumab in patients with $\mathrm{HeFH}$. The placebo-corrected reductions in LDL-C at week 12 were $59 \%$ for evolocumab $140 \mathrm{mg}$ every 2 weeks and $61 \%$ for evolocumab 420 mg every month. Patients in the evolocumab groups also exhibited significant reductions in apoB (49\%), Lp(a) (about $30 \%$ ), nonHDL cholesterol (non-HDLc) (55\%), and TG (10-20\%) and increases in HDLc $(9 \%)^{28}$.

\section{Patients with homozygous familial} hypercholesterolemia (HoFH)

50 patients diagnosed with $\mathrm{HoFH}$ and high levels of LDL-C despite appropriate lipid-lowering therapy received evolocumab $420 \mathrm{mg}$ or placebo every 4 weeks for 12 weeks. Patients in the treatment group had significant reductions in LDL-C (31\%) and apoB (23\%) compared to placebo. Meanwhile, changes in $\operatorname{Lp}(a)$, HDLC, and TG did not differ significantly between groups $^{29}$. In a longer timeframe, TAUSSIG was an open-label, non-randomized trial that studied evolocumab in 106 patients with HoFH over 48 weeks. Patients received $420 \mathrm{mg}$ of evolocumab monthly or biweekly if on LDL apheresis. The LDL-C reduction at week 48 compared to baseline was $23.3 \%$, with simultaneous reductions of $16.2 \%$ in apoB and $11.9 \%$ in $\mathrm{Lp}(\mathrm{a})$, and a $7.6 \%$ increase in $\mathrm{HDLc}^{30}$.

\section{Patients with common hypercholesterolemia}

The efficacy of evolocumab monotherapy in common (not monogenic) hypercholesterolemia was assessed in MENDEL-2. This was a multicenter trial of 614 patients with LDL-C above $100 \mathrm{mg} / \mathrm{dL}$, and 10-year Framingham risk score estimated CVD risk under $10 \%$, not on statin treatment. In à doubleplacebo design, patients were randomized to receive evolocumab $140 \mathrm{mg}$ biweekly, evolocumab $420 \mathrm{mg}$ monthly, and ezetimibe $10 \mathrm{mg}$ daily or placebo. After 12 weeks, biweekly evolocumab reduced LDL-C by $57 \%$, and for monthly evolocumab, the reduction was $56 \%^{31}$.

\section{Patients who are receiving a statin}

The LAPLACE trial evaluated the efficacy of evolocumab $140 \mathrm{mg}$ biweekly, evolocumab $420 \mathrm{mg}$ monthly, and ezetimibe or placebo when added to high-intensity (atorvastatin $80 \mathrm{mg}$ or rosuvastatin $40 \mathrm{mg}$ ) or moderate-intensity (atorvastatin $10 \mathrm{mg}$, rosuvastatin $5 \mathrm{mg}$ or simvastatin $40 \mathrm{mg}$ ) statin therapy. After 12 weeks, evolocumab reduced LDL-C by $66-75 \%$ in the moderate statin group, and by $63-75 \%$ in the high-intensity statin group (both compared with placebo) $)^{32}$.

\section{Patients outside LDL-C goal with different CVD risk profiles}

The most common scenario for considering the use of a PCSK9 inhibitor is in patients who do not achieve LDL-C goals despite the use of other currently available therapies. The DESCARTES trial addressed the efficacy of evolocumab in this situation. Patients outside their LDL-C goal according to the Adult Treatment Panel III guidelines were given lipid-lowering treatment for 4-12 weeks with diet alone, diet plus 
atorvastatin $10 \mathrm{mg}$, atorvastatin $80 \mathrm{mg}$ daily alone, or atorvastatin $80 \mathrm{mg}$ plus ezetimibe $10 \mathrm{mg}$. The 905 patients who still had an LDL-C above $75 \mathrm{mg} / \mathrm{dL}$ were randomized to monthly evolocumab $420 \mathrm{mg}$ or placebo. After 52 weeks, evolocumab reduced LDL-C between $48 \%$ (atorvastatin $80+$ ezetimibe group) and $62 \%$ (diet alone group) ${ }^{33}$.

\section{Patients with statin intolerance}

Use of evolocumab versus ezetimibe in this particular group was studied in the GAUSS- 3 trial, which enrolled 511 patients with high LDL-C, who could not tolerate effective doses of at least two different statins due to muscle-related side effects. The reduction in LDL-C at week 24 for monthly evolocumab $420 \mathrm{mg}$ (versus ezetimibe) was $36 \%^{34}$.

\section{IMPACT OF EVOLOCUMAB ON HARD CVD OUTCOMES}

OSLER 1 and 2 were long-term extension trials of patients who had completed either a phase 2 or phase 3 clinical trial with evolocumab in a background of standard therapy. The main purpose of the study was to examine the long-term safety of evolocumab, but adverse CVD events were also recorded and analyzed in an exploratory way. After a median follow-up of 11 months, the rate of CVD events was significantly lower in the evolocumab group compared to placebo (HR: $0.47,95 \% \mathrm{Cl}$ : 0.28-0.78). OSLER also confirmed the findings from Phase II studies regarding the lipid efficacy of evolocumab on LDL-C, non-HDLC, apoB, TG, and $L p(a)^{35}$. FOURIER was the pivotal, CVD outcomes trial of evolocumab. More than 27,000 patients with clinically evident atherosclerotic CVD and LDL-C above $70 \mathrm{mg} / \mathrm{dL}$ despite statin therapy was randomized to biweekly evolocumab $140 \mathrm{mg}$, monthly evolocumab 420, or matching placebo. Evolocumab significantly reduced the risk of the primary endpoint (cardiovascular death, myocardial infarction, stroke, hospitalization for unstable angina, or coronary revascularization) (HR: $0.85,95 \% \mathrm{Cl}: 0.79-0.92$ ) and of a secondary endpoint of cardiovascular death, myocardial infarction, or stroke (HR: $0.80,95 \% \mathrm{Cl}: 0.73-0.88)^{36}$.

\section{ADVERSE EVENTS WITH USE OF EVOLOCUMAB}

OSLER and FOURIER provided reassuring results concerning the long-term safety of this type of agents. The most frequent adverse events were injection site reactions ( $4.3 \%$ in OSLER, $2.1 \%$ in FOURIER) ${ }^{35,36}$. In FOURIER, evolocumab-binding antibodies were equally prevalent at the end of follow-up in the evolocumab and standard-therapy groups (0.3\%). Since PCSK9 inhibitors take LDL-C to values seldom reached with other agents, there has been some concern about potentially adverse neurocognitive consequences of these very LDL-C concentrations. EBBINGHAUS was a substudy of FOURIER, in which formal neurocognitive assessments were administered to patients in the evolocumab and placebo groups of FOURIER (586 evolocumab and 618 placebo patients) ${ }^{37}$. Changes in neurocognitive scores were numerically very similar and statistically not different between patients in the evolocumab and placebo groups. The same results were observed for the spatial working memory strategy index of executive function (primary outcome), working memory, episodic memory, and psychomotor speed.

\section{Alirocumab}

Alirocumab is a human mAb of the IgG1 isotype, directed against PCSK9. The clinical development program for alirocumab received the name ODYSSEY.

\section{EFFICACY OF ALIROCUMAB}

\section{Patients with $\mathrm{HeFH}$}

Use of alirocumab in patients with $\mathrm{HeFH}$ was tested in the ODYSSEY FH-I and ODYSSEY FH-II trials ${ }^{38}$. A total of 735 patients with $\mathrm{HeFH}$ and inadequate LDL$\mathrm{C}$ control on maximally tolerated lipid-lowering therapy were randomized to biweekly alirocumab $75 \mathrm{mg}$ or placebo, with forced titration to biweekly $150 \mathrm{mg}$ if LDL-C was $>70 \mathrm{mg} / \mathrm{dL}$ at week 8. At week 24 , LDL-C in the alirocumab group decreased $51-58 \%$ versus placebo; the reductions persisted over 78 weeks of follow-up. Similar to evolocumab, significant positive changes were observed in apoB, $L p(a), T G$, and HDLc. Some patients with particularly severe forms of $\mathrm{HeFH}$ required $\mathrm{LDL}$ apheresis to control their LDL-C levels. The ODYSSEY ESCAPE trial assessed whether the use of alirocumab could eliminate the requirement for $L D L$ apheresis in such patients. Sixtytwo patients undergoing regular weekly or biweekly lipoprotein apheresis were randomly assigned to receive alirocumab $150 \mathrm{mg}$ or placebo every 2 weeks for 18 weeks. During weeks 7-18 of the study, the apheresis rate was adjusted based on the LDL-C of 
each patient; if the $L D L-C$ reduction was > $30 \%$, then apheresis was suspended. Alirocumab-treated patients had a $75 \%$ reduction in the standardized rate of apheresis treatments versus placebo ${ }^{39}$.

\section{Patients who are receiving a statin}

Two studies tested the efficacy of alirocumab when combined with maximally tolerated doses of statins, the ODYSSEY COMBO-I and ODYSSEY COMBO-II trials. The difference between the two resides in the comparator: Placebo in ODYSSEY COMBO-I and ezetimibe in ODYSSEY COMBO-II ${ }^{40}$. The placebo-subtracted change in LDL-C with alirocumab was $46 \%$, even though more than $80 \%$ of patients in the alirocumab group had remained on the $75 \mathrm{mg}$ biweekly dose. ODYSSEY COMBO-II followed a similar design, but with a longer follow-up (52 weeks), active comparator (ezetimibe), and larger sample size (720 patients). Reductions in LDL-C from baseline were $51 \%$ for alirocumab and $21 \%$ for ezetimibe. These changes were maintained over the 52 -week follow-up ${ }^{41}$.

\section{Patients outside LDL-C goal with different CVD risk profiles}

A set of studies compared alirocumab with other alternatives aimed at bringing patients into their riskdefined LDL-C goal. Alirocumab proved to be the strategy with the highest probability of bringing patients to goal: $85-90 \%$ of all patients on alirocumab reached and maintained their $\mathrm{LDL}-\mathrm{C}$ levels within goal, compared to $65-70 \%$ with ezetimibe, $10-35 \%$ with doubling of atorvastatin, and $40-65 \%$ with switching to rosuvastatin $40 \mathrm{mg} / \mathrm{d}^{42}$.

\section{Patients with statin intolerance}

ODYSSEY ALTERNATIVE was a randomized trial focused on the comparative efficacy of alirocumab versus ezetimibe in patients at moderate-to-high cardiovascular risk who could not tolerate at least two different statins ${ }^{43}$. After a statin rechallenge to confirm intolerance, 361 patients received alirocumab $75 \mathrm{mg}$ every 2 weeks + daily oral placebo, ezetimibe $10 \mathrm{mg} / \mathrm{d}$ + biweekly injected placebo, or atorvastatin $20 \mathrm{mg} / \mathrm{d}+$ biweekly injected placebo (rechallenge strategy). Alirocumab reduced LDL-C by $45 \%$, compared to only $15 \%$ for ezetimibe. Muscular symptoms were significantly less frequent with alirocumab compared to the statin rechallenge group (HR: 0.61, 95\% Cl: 0.-0.99).

\section{IMPACT OF ALIROCUMAB ON HARD CVD OUTCOMES}

The first study to examine (albeit as a secondary analysis) the impact of alirocumab on CVD events was ODYSSEY LONG TERM ${ }^{44}$. In an observational post hoc analysis, the rate of CVD events (death from CHD, nonfatal myocardial infarction, fatal or nonfatal ischemic stroke, or unstable angina requiring hospitalization) was significantly lower with alirocumab (HR: $0.52,95 \% \mathrm{Cl}: 0.31-0.90$ ). The pivotal CVD outcomes trial of alirocumab is ODYSSEY OUTCOMES $^{45}$. More than 18,000 patients with a recent acute coronary syndrome who already received maximally tolerated doses of statins were randomized to alirocumab every 2 weeks or placebo. In the alirocum$\mathrm{ab}$ arm, the dose was titrated aiming to keep LDL-C between 25 and $50 \mathrm{mg} / \mathrm{dL}$, but above $15 \mathrm{mg} / \mathrm{dL}$. After 48 months, major adverse cardiac events occurred in $9.5 \%$ of patients in the alirocumab group and $11.1 \%$ of patients within the placebo group (HR: 0.85 , $95 \% \mathrm{Cl}: 0.78-0.93$ ). This impact took place mostly at the expense of a reduction in total myocardial infarction and ischemic stroke. A somewhat puzzling finding was a significant $0.6 \%$ absolute risk reduction on allcause mortality, but with no effect on cardiovascular mortality.

\section{ADVERSE EVENTS WITH USE OF ALIROCUMAB}

The highest rate of injection-site reactions with alirocumab was observed in the ODYSSEY $\mathrm{FH}$ trials ( $12.4 \%$ in $\mathrm{FH} \mathrm{I}$ and $11.4 \%$ in $\mathrm{FH} \mathrm{II),} \mathrm{but} \mathrm{similar} \mathrm{rates}$ were observed with placebo, $(11.0 \%$ and $7.4 \%$, respectively $)^{38,44}$. ODYSSEY Outcomes found reassuringly low rates of adverse events with the continued use of alirocumab over 4 years. The only concerning finding was a high rate of development of neutralizing anti-drug antibodies in the alirocumab group (42\%), while in the placebo group, only $6 \%$ of participants developed such antibodies. These antibodies slightly affected the lipid efficacy of the agent, but apparently did not influence its impact on CVD risk.

\section{Bococizumab}

Bococizumab is a humanized (not fully human) antibody against PCSK9, initially developed by Pfizer. Clinical development of bococizumab was halted in 
2016 after results of the 10-month SPIRE trial showed a high rate of development of neutralizing anti-drug antibodies, progressive loss of efficacy, and no impact on CVD outcomes ${ }^{46}$.

\section{LY3015014}

LY3015014 is a novel humanized mAb of the IgG4 isotype, directed against PCSK9. It has a somewhat different mechanism of action as it allows for the catalytic cleavage of PCSK9, thus reducing the plasma concentrations of intact PCSK947. The IgG4 isotype also confers LY3015014 a longer half-life, making administration every 8 weeks possible. In a 16-week, phase II study of patients with primary hypercholesterolemia ${ }^{48}$, LY3015014 achieved dose-dependent placebo-corrected LDL-C reductions between 22 and $58 \%$, with a maximal reduction observed for the 300 $\mathrm{mg}$ monthly dose. No relevant adverse events were reported.

\section{mAb against ANGPTL3}

\section{Evinacumab}

Evinacumab is a fully human monoclonal antibody of the IgG4 isotype against ANGPTL3. The hinge region of the IgG contains a stabilizing mutation to protect it from forming half-antibodies ${ }^{49}$. In a proof-of-concept study in 83 patients with LDL-C above $100 \mathrm{mg} /$ $\mathrm{dL}$ and/or plasma TG between 150 and $450 \mathrm{mg} / \mathrm{dL}$, evinacumab $20 \mathrm{mg} / \mathrm{kg}$ administered intravenously induced a $76 \%$ reduction in plasma TG, a $23 \%$ reduction in LDL-C, and a $18 \%$ reduction in HDLc. A single subcutaneous dose of evinacumab induced a $64 \%$ reduction in TG after 4 days. With subcutaneous administration, the effects had disappeared by day 15 , but with intravenous administration, the effects persisted up to day $15^{16}$. The most common adverse event with evinacumab was headache in about $10 \%$ of patients.

In a group of nine patients with HoFH who were already receiving aggressive lipid-lowering regimens with statins, ezetimibe, lomitapide and/or PCSK9 mAbs, evinacumab induced remarkable benefits ${ }^{50}$. Patients achieved reductions between 25\% and $90 \%$ in LDL-C, with great interindividual variability depending on the type of LDLr mutation (null vs. non-null, homozygous vs. compound heterozygous). Larger and longer clinical trials with evinacumab are currently in course to test the efficacy of this $m A b$ in diverse patient populations.

\section{Antisense oligonucleotides}

ASO are single-stranded nucleic acid molecules (mostly DNA or modified DNA) that bind through Watson-Crick complementarity to cellular mRNAs and induce their degradation or prevent their translation. In doing so, ASO reduce the cellular synthesis of a target protein. ASO are usually 12-20 nucleotides long, antisense, and complementary to the sense sequence of the target mRNA. Multiple chemical modifications can be introduced into the sugar-phosphate backbone of ASO to prolong their stability, improve cellular uptake, or reduce susceptibility to attack by extracellular nucleases ${ }^{51,52}$. Once inside the cell, ASO bind specifically to regions of the target mRNA forming a DNA-RNA heteroduplex. This complex recruits RNase $\mathrm{H} 1$, an endonuclease that cleaves the RNA strand $^{53}$. ASO-based therapies accumulate in the liver where most of the apo are produced, bind specifically to their target mRNAs and are metabolized by a pathway completely independent of the cytochrome P450. These properties theoretically grant them a lower risk of adverse events or pharmacological interactions $^{54}$. First-generation ASO were created by replacing one of the non-bridging oxygens of the phosphate group in DNA with sulfur to impede nuclease activity, but they possessed cellular toxicity at high doses $^{55}$. Second-generation ASO have modified ribose with a methyl or methoxyethyl group in their 2' Carbon. Mixed modifications that combine changes in the ribose and the phosphate groups attenuate enzymatic degradation and protein binding and enhance affinity to RNA and the endonucleases ${ }^{56}$.

\section{Mipomersen}

Mipomersen is a 20 nucleotide long, second-generation ASO directed against the mRNA for apoB. Mipomersen is currently approved only for the orphan indication of $\mathrm{HoFH}$. The key trial in securing the regulatory approval of mipomersen recruited 51 patients with $\mathrm{HoFH}$ who were on intensive lipid-lowering therapy excluding lipoprotein apheresis ${ }^{57}$. Twenty-five patients in the mipomersen group and 18 in the placebo group completed the 26-week follow-up. 
Mipomersen achieved a somewhat modest reduction of LDL-C by $21 \%$ with a concomitant reduction of $24 \%$ in apoB. The main caveat, however, was the huge interindividual variability in response to the agent, with changes in LDL-C vs. baseline ranging from $+2 \%$ to $-83 \%$. Studies in $\mathrm{HeFH}$ patients have found similarly wide variations, with individual $\mathrm{LDL}-\mathrm{C}$ responses ranging from +30 to $-70 \%$ versus baseline ${ }^{58}$. The most common adverse effects of mipomersen, when used continuously for 2 years, are injection-site reactions ( $98 \%$ of patients), flu-like symptoms $(65 \%)$, and clinically relevant elevations of liver enzymes $(13 \%)^{59}$. Most studies have also documented significant increases in hepatic fat content (in line with the drug's mechanism -inhibition of lipoprotein production and secretion by the liver) ${ }^{3,57,58}$. A study of liver biopsies from patients chronically treated with mipomersen showed severe steatosis in most patients (five out of seven), albeit without histological signs of nonalcoholic steatohepatitis. No dedicated studies have been undertaken to evaluate the impact of LDL$C$ with mipomersen on CVD outcomes, but a pooled analysis that included 233 patients (most of them with familial hypercholesterolemia) from 3 trials lasting 1 year or longer ${ }^{60}$ tried to address this question. The rate of CVD events during the 24 months before randomization in the mipomersen group was compared to MACE after the initiation of mipomersen. CVD events occurred in $9.6 \%$ of patients during the 24 months posterior to mipomersen initiation, compared to $61.5 \%$ during the 24 months before mipomersen treatment. Thus, mipomersen use was associated with $95 \%$ lower odds of CVD events.

\section{IONIS-ANGPTL3-LRx}

IONIS-ANGPTL3-LRx is a second-generation, $\mathrm{N}$-acetyl galactosamine-conjugated ASO against ANGPTL3. A proof-of-concept study assessed IONIS-ANGPTL3LRx in 44 participants with plasma TG $90-150 \mathrm{mg} /$ $\mathrm{dL}$ (low-dose group) or $>150 \mathrm{mg} / \mathrm{dL}$ (high-dose group). Patients received 10, 20, 40, or $60 \mathrm{mg}$ of IONIS-ANGPTL3-LRx or placebo every week for 6 weeks. Patients in the active treatment groups had reductions between $33 \%$ and $63 \%$ in plasma TG, between $1 \%$ and $33 \%$ in LDL-C, and between $10 \%$ and $37 \%$ in non-HDLc. ApoB and apoC-III were also significantly reduced. No serious adverse events were observed ${ }^{61}$.

\section{Volanesorsen}

Volanesorsen (or ISIS 304801) is a second-generation ASO complementary to the APOC 3 mRNA. This ASO blocks hepatic apoC-III production and secretion, improving removal of circulating atherogenic lipoproteins.

A phase II study tested volanesorsen in patients with untreated severe hypertriglyceridemia (plasma TG $350-2,000 \mathrm{mg} / \mathrm{dL}, 41$ received volanesorsen and 16 placebo), and in patients treated with a stable dose of a fibrate and with plasma TG $225-2,000 \mathrm{mg} / \mathrm{dL}$ (20 received volanesorsen and 8 placebo) ${ }^{62}$. Volanesorsen 100-300 mg per week induced dose-dependent reductions in plasma apoC-III over 13 weeks, which translated into a $31.3-70.9 \%$ drop in fasting plasma TG. Response to the agent was considerably consistent across all doses, in contrast to the large interindividual variability observed with other ASO. In a pilot study in patients with type 2 diabetes and hypertriglyceridemia ${ }^{63}, 15$ patients with type 2 diabetes and plasma TG between 200 and $500 \mathrm{mg} / \mathrm{dL}$ were randomized 2:1 to receive volanesorsen $300 \mathrm{mg}$ or placebo every week for 15 weeks. The impressive drop in plasma apoC-III (88\%) was accompanied by a $69 \%$ reduction in plasma TG and a $42 \%$ increase in HDLc versus placebo. Moreover, clamp-measured whole-body insulin sensitivity improved by $57 \%$ in the volanesorsen group. In a small, but relevant study of three patients with familial chylomicronemia, volanesorsen proved to be similarly effective, inducing profound suppression of apoC-III synthesis and TG reductions between $56 \%$ and $86 \% 64$.

\section{ASO against apo (a)}

\section{ISIS-APO(a)Rx}

ISIS-APO(a)Rx is a specific ASO that specifically binds a splice site between exons 24 and 25 of the apo(a) mRNA. The agent was selected from a screen of over 2200 ASO complementary to multiple sites across the human apo(a) transcript ${ }^{65}$. In a phase 1 study on healthy volunteers, ISIS-APO(a) Rx 100-300 mg reduced plasma $L p(a)$ by $40-78 \%{ }^{66}$.

\section{IONIS-APO(a)- $L_{R x}$}

IONIS-APO(a)-LRx is a ligand-conjugated ASO with a triantennary $\mathrm{N}$-acetylgalactosamine complex 
covalently attached to allow faster and specific uptake by hepatocytes. In a phase $1 / 2$ a trial in healthy volunteers with $L p(a)>75 \mathrm{nmol} / \mathrm{L}$, multiple doses of 10-40 mg IONIS-APO(a)LRx administered at days 1 , $3,5,8,15$, and 22 induced $L p(a)$ reductions between $66 \%$ and $92 \%$ at day $36^{67}$. Isolated case reports suggest that knocking down $\mathrm{Lp}(\mathrm{a})$ may have a large clinical impact ${ }^{65}$, but longer and larger clinical trials will be required to confirm this hypothesis.

\section{SMALL, INTERFERING RNAS (siRNAs)}

siRNAs are part of the larger family of regulatory RNAs. Specifically, siRNAs are synthetic, doublestranded RNAs that can induce RNAi (RNA interference $)^{68}$. RNAi is a mechanism used by cells to defend themselves against foreign or invasive nucleic acids and to aid to the maintenance of genome integrity. When an unknown dsRNA enters the cell, it is attacked by Dicer, an RNAse III endonuclease ${ }^{69}$, giving rise to 21-23 bp-long dsRNA molecules with 3'overhangs (siRNAs). SiRNAs are then loaded onto the RNA-induced silencing complex (RISC), formed by the siRNA, Dicer and the protein Argonaute2 (Ago 2$)^{54}$. Once the RISC is formed, one of the strands (passenger strand) is degraded, and the other (guide strand) is preserved in the complex. The guide strand in RISC binds the target mRNA through Watson-Crick complementarity and leads to its cleavage by Dicer $^{70}$, potentially generating more siRNAs. Through this amplification mechanism, a few siRNAs are able to silence expression of a gene over long periods of time. Therapeutic siRNAs can be chemically modified to decrease susceptibility to endo- and exonucleases, avoid rapid renal excretion, and increase their tissue specificity ${ }^{71}$. The internalization of therapeutic siRNAs is achieved by endocytosis due to their negative charge and large molecular weight ${ }^{72}$.

\section{Inclisiran}

Inclisiran is a synthetic siRNA directed against PCSK 973. ORION-1 was a dose-finding trial of subcutaneous inclisiran vs. placebo in patients with high CVD risk and high LDL-C despite maximally tolerated statin. Patients had LDL-C concentrations above $70 \mathrm{mg} / \mathrm{dL}$ if they had a history of CVD or above $100 \mathrm{mg} / \mathrm{dL}$ if they did not have such history. Inclisiran was tested at doses ranging from 200 to
$500 \mathrm{mg}$, and as a single dose at day 1 or 2 doses at days 1 and 90 . Plasma LDL-C in day 180 declined by $27.9-41.9 \%$ in the single-dose group and by $35.5-52.6 \%$ in the two-doses groups. Interestingly, plasma PCSK9 and LDL-C still remained significantly below their baseline levels at day 240. The most common adverse event was injection-site reactions, which happened in $5 \%$ of patients on inclisiran. In contrast to studies with ASO-based therapies, no flu-like symptoms were reported in the active treatment groups.

\section{THE FUTURE: CRISPR/CRISPR- ASSOCIATED 9 (CAS9)-BASED INVESTIGATIONAL THERAPIES}

CRISPR-Cas9 systems originated as a primitive immunity mechanism displayed by some bacterial species. Since the year 2013, they have been harnessed as biotechnological tools for genome editing ${ }^{74}$ in eukaryotic cells and in vivo. These systems offer the attractive possibility of "knocking off" or even "switching on" a specific gene in a complete, live multicellular organism.

Synthetic CRISPR-Cas9 systems comprise a protein (Cas9) and an RNA molecule. Cas9 is an enzyme with helicase activity, selective DNA binding activity, and endonuclease activity ${ }^{75}$. The RNA component is called a "guide RNA," it is about 100 nucleotides long and provides the CRISPR-Cas9 complex with sequence specificity. After binding Cas9, the guide RNA hybridizes to one strand of dsDNA through a so-called "protospacer" sequence contained within its first 20 nucleotides. Meanwhile, Cas9 binds a much shorter sequence in the DNA, adjacent to the protospacer. This protospacer-adjacent motif is different in each type of Cas9 protein. Under this configuration, a Cas9/guide RNA/target DNA complex is formed, and a ds break is introduced in the DNA. In this fashion, systems with the ability to introduce breaks at specific points in virtually any site of a genome can be designed ${ }^{76}$. The most commonly used versions of Cas9 are those from Streptococcus pyogenes and Staphylococcus aureus ${ }^{75}$.

The versatility of these systems brings about very interesting applications collectively termed "epigenome editing." If Cas9 is made catalytically inactive 
and fused into a chimeric protein with a transcriptional activator domain, the expression of a target gene can be stimulated (CRISPR activation). If catalytically inactive Cas9 is fused to a transcriptional repressor, the result is gene silencing at the transcriptional level (CRISPR interference) ${ }^{77}$. In CRISPRCas9-based base editing, Cas9 is fused with a cytosine deaminase domain, resulting in a $\mathrm{C}-$ to- $\mathrm{U}$ change at the desired place (with the possibility of introducing a stop codon) or in a G-to-A change in the opposite DNA strand through cellular DNA repair systems.

The main concerns with the therapeutic use of CRISPR-Cas9 systems have to do with the potential introduction of unintended changes at genomic sites different from the target and with the choice of an appropriate vector that guarantees efficient delivery of RNAs encoding both the guide RNA and Cas9 (adeno-associated viral vectors and lipid nanoparticles have been attempted) $)^{75}$.

A proof-of-concept study in mice demonstrated that an S. aureus CRISPR-Cas9 system targeted to break and disrupt the PCSK9 gene could reduce plasma PCSK 9 by more than $90 \%$ and reduce plasma cholesterol by about $40 \%$, without signs of off-target mutagenesis or liver toxicity ${ }^{78}$. Similar results were found with an S. pyogenes CRISPR-Cas9 base edition strategy in adult mice ${ }^{79}$. More recently, an in vitro screen for potential sites amenable to introduction of baseedited nonsense mutations in ANGPTL3 identified a suitable site at codon Gln-135 of the gene. Researchers produced adenoviral vectors containing a CRISPRCas 9 base editor with a guide RNA targeting Gln-135 of ANGPTL3 and injected them into 5-week-old mice. 7 days after injection of the adenoviruses, plasma ANGPTL 3 was reduced by $49 \%$, plasma TG by $31 \%$, and plasma cholesterol by $19 \%{ }^{80}$. Deep sequencing of liver biopsies revealed no evidence of DNA edition at the top 10 most likely off-target sites. Injection of the same adenovirus into LDLr knockout mice (an animal model of $\mathrm{HoFH}$ ) reduced TG by $56 \%$ and total cholesterol by $51 \%$.

These results have created a great deal of expectations about the prospects for gene editing therapies, but major technical and safety hurdles will need to be overcome before they may become feasible options in the routine management of lipid disorders.
In summary, great advances in the physiology of lipoprotein metabolism have translated into the discovery of new and relevant targets for inhibition. Molecular biology tools aimed at these targets have provided a vast arsenal for the treatment of lipid disorders includes that now includes mAb, ASO, siRNAs and sometime in the future perhaps CRISPR/CAS9-based agents. All these advances will enhance our ability to prevent CVD and improve the lifes of our patients.

\section{REFERENCES}

1. Bruzzone G, Corbelli G, Belci P, et al. Cholesterol lowering therapy: treat to target or reduce the global risk? The unresolved problem of residual risk. Curr Pharm Des. 2016;22:5676-86.

2. Turner RM, Yin P, Hanson A, et al. Investigating the prevalence, predictors, and prognosis of suboptimal statin use early after a non-ST elevation acute coronary syndrome. J Clin Lipidol. 2017; 11:204-14.

3. Ajufo E, Rader DJ. New therapeutic approaches for familial hypercholesterolemia. Annu Rev Med. 2018;69:113-31.

4. Chait A, Eckel RH. Lipids, lipoproteins, and cardiovascular disease: clinical pharmacology now and in the future.] Clin Endocrinol Metab. 2016;101:804-14.

5. Abifadel $M$, Varret M, Rabès JP, et al. Mutations in PCSK9 cause autosomal dominant hypercholesterolemia. Nat Genet. 2003, 34:154-6.

6. Seidah NG, Benjannet S, Wickham L, et al. The secretory proprotein convertase neural apoptosis-regulated convertase 1 (NARC-1): liver regeneration and neuronal differentiation. Proc Natl Acad Sci U S A. 2003;100:928-33.

7. Maxwell KN, Breslow JL. Adenoviral-mediated expression of Pcsk 9 in mice results in a low-density lipoprotein receptor knockout phenotype. Proc Natl Acad Sci U S A. 2004;101:7100-5.

8. Cohen J, Pertsemlidis A, Kotowski IK, et al. Low LDE cholesterol in individuals of African descent resulting from frequent nonsense mutations in PCSK9. Nat Genet. 2005;37:161-5.

9. Cohen JC, Boerwinkle E, Mosley TH Jr., Hobbs HH. Sequence variations in PCSK9, low LDL, and protection against coronary heart disease. N Engl J Med. 2006;354:1264-72.

10. Kathiresan S, Myocardial Infarction Genetics Consortium. A PCSK9 missense variant associated with a reduced risk of earlyonset myocardial infarction. N Engl J Med. 2008;358:2299-300.

11. Zhang DW, Lagace TA, Garuti R, et al. Binding of proprotein convertase subtilisin/kexin type 9 to epidermal growth factorlike repeat $A$ of low density lipoprotein receptor decreases receptor recycling and increases degradation. J Biol Chem. 2007; 282:18602-12.

12. Mattijssen F, Kersten S. Regulation of triglyceride metabolism by angiopoietin-like proteins. Biochim Biophys Acta. 2012;1821: $782-9$.

13. Koishi R, Ando $Y$, Ono M, et al. Angptl3 regulates lipid metabolism in mice. Nat Genet. 2002;30:151-7.

14. Haller JF, Mintah IJ, Shihanian LM, et al. ANGPTL8 requires ANGPTL3 to inhibit lipoprotein lipase and plasma triglyceride clearance. J Lipid Res. 2017;58:1166-73.

15. Stitziel NO, Khera AV, Wang $X$, et al. ANGPTL3 deficiency and protection against coronary artery disease. J Am Coll Cardiol. 2017;69:2054-63.

16. Dewey FE, Gusarova V, Dunbar RL, et al. Genetic and pharmacologic inactivation of ANGPTL3 and cardiovascular disease. N Engl J Med. 2017;377:211-21.

17. Robciuc MR, Maranghi M, Lahikainen A, et al. Angptl3 deficiency is associated with increased insulin sensitivity, lipoprotein lipase activity, and decreased serum free fatty acids. Arterioscler Thromb Vasc Biol. 2013;33:1706-13.

18. Mendivil CO, Zheng C, Furtado J, et al. Metabolism of very-lowdensity lipoprotein and low-density lipoprotein containing apolipoprotein C-III and not other small apolipoproteins. Arterioscler Thromb Vasc Biol. 2010;30:239-45.

19. Mendivil CO, Rimm EB, Furtado J, Sacks FM. Apolipoprotein E in VLDL and LDL with apolipoprotein C-III is associated with a lower risk of coronary heart disease. J Am Heart Assoc. 2013; 2:e000130. 
20. Mendivil CO, Rimm EB, Furtado J, Chiuve SE, Sacks FM. Lowdensity lipoproteins containing apolipoprotein C-III and the risk of coronary heart disease. Circulation. 2011;124:2065-72.

21. Jensen MK, Rimm EB, Furtado JD, Sacks FM. Apolipoprotein C-III as a potential modulator of the association between HDL-cholesterol and incident coronary heart disease. J Am Heart Assoc. 2012;1:jah3-e000232.

22. Pollin TI, Damcott $\mathrm{CM}$, Shen $\mathrm{H}$, et al. A null mutation in human APOC 3 confers a favorable plasma lipid profile and apparent cardioprotection. Science. 2008;322:1702-5

23. Maranhão RC, Carvalho PO, Strunz CC, Pileggi F. Lipoprotein (a): structure, pathophysiology and clinical implications. Arq Bras Cardiol. 2014;103:76-84.

24. Emerging Risk Factors Collaboration, Erqou S, Kaptoge S, et al. Lipoprotein(a) concentration and the risk of coronary heart disease, stroke, and nonvascular mortality. JAMA. 2009;302:412-23.

25. Clarke R, Peden JF, Hopewell JC, et al. Genetic variants associated with Ip(a) lipoprotein level and coronary disease. N Engl J Med. 2009;361:2518-28.

26. Köhler G, Milstein C. Continuous cultures of fused cells secreting antibody of predefined specificity. Nature. 1975;256:495-7.

27. Stoekenbroek RM, Kastelein JJ, Huijgen R. PCSK9 inhibition: the way forward in the treatment of dyslipidemia. BMC Med. 2015; 13:258.

28. Raal FJ, Stein EA, Dufour R, et al. PCSK9 inhibition with evolocumab (AMG 145) in heterozygous familial hypercholesterolaemia (RUTHERFORD-2): a randomised, double-blind, placebocontrolled trial. Lancet. 2015;385:331-40.

29. Raal FJ, Honarpour N, Blom DJ, et al. Inhibition of PCSK9 with evolocumab in homozygous familial hypercholesterolaemia (TESLA part B): A randomised, double-blind, placebo-controlled trial. Lancet. 2015;385:341-50.

30. Raal FJ, Hovingh GK, Blom D, et al. Long-term treatment with evolocumab added to conventional drug therapy, with or without apheresis, in patients with homozygous familial hypercholesterolemia: an interim subset analysis of the open-label TAUSSIG study. Lancet Diabetes Endocrinol. 2017;5:280-90.

31. Koren MJ, Lundqvist P, Bolognese M, et al. Anti-PCSK9 monotherapy for hypercholesterolemia: the MENDEL-2 randomized, controlled phase III clinical trial of evolocumab. J Am Coll Cardiol. 2014;63:2531-40.

32. Robinson JG, Nedergaard BS, Rogers WJ, et al. Effect of evolocumab or ezetimibe added to moderate-or high-intensity statin therapy on LDL-C lowering in patients with hypercholesterolemia. JAMA. 2014;311:1870.

33. Blom DJ, Hala T, Bolognese $M$, et al. A 52-week placebo-controlled trial of evolocumab in hyperlipidemia. $N$ Engl J Med. 2014;370:1809-19.

34. Nissen SE, Stroes E, Dent-Acosta RE, et al. Efficacy and tolerability of evolocumab vs ezetimibe in patients with muscle-related statin intolerance: the GAUSS-3 randomized clinical trial. JAMA. 2016:315:1580-90

35. Sabatine MS, Giugliano RP, Wiviott SD, et al. Efficacy and safety of evolocumab in reducing lipids and cardiovascular events. N Engl J Med. 2015;372:1500-9.

36. Sabatine MS, Giugliano RP, Keech AC, et al. Evolocumab and clinical outcomes in patients with cardiovascular disease. N Engl J Med. 2017;376:1713-22.

37. Giugliano RP, Mach F, Zavitz K, et al. Cognitive function in a randomized trial of evolocumab. N Engl J Med. 2017;377:633-43.

38. Kastelein JJ, Ginsberg HN, Langslet G, et al. ODYSSEY FH I and FH II: 78 week results with alirocumab treatment in 735 patients with heterozygous familial hypercholesterolaemia. Eur Heart J. 2015;36:2996-3003.

39. Moriarty PM, Parhofer KG, Babirak SP, et al. Alirocumab in patients with heterozygous familial hypercholesterolaemia undergoing lipoprotein apheresis: the ODYSSEY ESCAPE trial. Eur Heart J. 2016;37:3588-95.

40. Kereiakes DJ, Robinson JG, Cannon CP, et al. Efficacy and safety of the proprotein convertase subtilisin/kexin type 9 inhibitor alirocumab among high cardiovascular risk patients on maximally tolerated statin therapy: the ODYSSEY COMBO I study. Am Heart J. 2015;169:906-15E+15.

41. Cannon CP, Cariou B, Blom D, et al. Efficacy and safety of alirocumab in high cardiovascular risk patients with inadequately controlled hypercholesterolaemia on maximally tolerated doses of statins: The ODYSSEY COMBO II randomized controlled trial. Eur Heart J. 2015;36:1186-94.

42. Bays H, Gaudet D, Weiss R, et al. Alirocumab as add-on to atorvastatin versus other lipid treatment strategies: ODYSSEY OPTIONS I randomized trial. J Clin Endocrinol Metab. 2015;100:3140-8.
43. Moriarty PM, Thompson PD, Cannon CP, et al. Efficacy and safety of alirocumab vs ezetimibe in statin-intolerant patients, with a statin rechallenge arm: the ODYSSEY ALTERNATIVE randomized trial. J Clin Lipidol. 2015;9:758-69.

44. Robinson JG, Farnier M, Krempf M, et al. Efficacy and safety of alirocumab in reducing lipids and cardiovascular events. $N$ Engl J Med. 2015;372:1489-99.

45. Steg $P$, The ODYSSEY OUTCOMES investigators. Presented at the American College of Cardiology Annual Scientific Session (ACC 2018), Orlando, FL; 2018. Available from: (accessed August 23, 2018)

46. Ridker PM, Revkin J, Amarenco P, et al. Cardiovascular efficacy and safety of bococizumab in high-risk patients. N Engl J Med. 2017:376:1527-39

47. Beyer TP, Eacho PI, Schroeder KM, et al. A PCSK9 antibody that blocks binding to LDLR while allowing normal PCSK 9 inactivation by furin is afforded a reduced clearance rate and a Tonger duration of effect in mice. Atheroscler Thromb Vasc Biol. 2015; 35:A538.

48. Kastelein JJ, Nissen SE, Rader DJ, et al. Safety and efficacy of LY3015014, a monoclonal antibody to proprotein convertase subtilisin/kexin type 9 (PCSK9): a randomized, placebo-controlled phase 2 study. Eur Heart J. 2016;37:1360-9.

49. Gusarova V, Alexa CA, Wang Y, et al. ANGPTL3 blockade with a human monoclonal antibody reduces plasma lipids in dyslipidemic mice and monkeys. J Lipid Res. 2015;56:1308-17.

50. Gaudet D, Gipe DA, Pordy R, et al. ANGPTL3 inhibition in homozygous familial hypercholesterolemia. N Engl J Med. 2017; 377:296-7

51. Miller CM, Harris EN. Antisense oligonucleotides: treatment strategies and cellular internalization. RNA Dis. 2016;3:e1393.

52. Toth PP. Antisense therapy and emerging applications for the management of dyslipidemia. J Clin Lipidol. 2011;5:441-9.

53. Crooke ST. Molecular mechanisms of antisense oligonucleotides. Nucleic Acid Ther. 2017:27:70-7

54. Besseling J, Hovingh GK, Stroes ES. Antisense oligonucleotides in the treatment of lipid disorders: pitfalls and promises. Neth J Med. 2013;71:118-22

55. Kurreck J. Antisense technologies. Improvement through nove chemical modifications. Eur ] Biochem. 2003;270:1628-44.

56. Agrawal S, Jiang Z, Zhao Q, et al. Mixed-backbone oligonucleotides as second generation antisense oligonucleotides: in vitro and in vivo studies. Proc Natl Acad Sci U S A. 1997:94:2620-5.

57. Raal FJ, Santos RD, Blom DJ, et al. Mipomersen, an apolipoprotein B synthesis inhibitor, for lowering of LDL cholesterol concentrations in patients with homozygous familial hypercholesterolaemia: a randomised, double-blind, placebo-controlled trial. Lancet. 2010;375:998-1006.

58. Stein EA, Dufour R, Gagne C, et al. Apolipoprotein B synthesis inhibition with mipomersen in heterozygous familial hypercholesterolemia: results of a randomized, double-blind, placebocontrolled trial to assess efficacy and safety as add-on therapy in patients with coronary artery disease. Circulation. 2012; 126:2283-92.

59. Santos RD, Duell PB, East C, et al. Long-term efficacy and safety of mipomersen in patients with familial hypercholesterolaemia: 2-year interim results of an open-label extension. Eur Heart J. 2015;36:566-75.

60. Duell PB, Santos RD, Kirwan BA, et al. Long-term mipomersen treatment is associated with a reduction in cardiovascular events in patients with familial hypercholesterolemia. J Clin Lipidol. 2016;10:1011-21.

61. Graham MJ, Lee RG, Brandt TA, et al Cardiovascular and metabolic effects of ANGPTL3 antisense oligonucleotides. N Engl ] Med. 2017;377:222-32.

62. Gaudet D, Alexander VJ, Baker BF, et al. Antisense inhibition of apolipoprotein C-III in patients with hypertriglyceridemia. N Engl J Med. 2015;373:438-47.

63. Digenio A, Dunbar RL, Alexander VJ, et al. Antisense-mediated lowering of plasma apolipoprotein C-III by volanesorsen improves dyslipidemia and insulin sensitivity in type 2 diabetes. Diabetes Care. 2016;39:1408-15

64. Gaudet D, Brisson D, Tremblay K, et al. Targeting APOC 3 in the familial chylomicronemia syndrome. N Engl ] Med. 2014; 371:2200-6

65. Graham MJ, Viney N, Crooke RM, Tsimikas S. Antisense inhibition of apolipoprotein (a) to lower plasma lipoprotein (a) levels in humans. J Lipid Res. 2016;57:340-51.

66. Tsimikas S, Viney NJ, Hughes SG, et al. Antisense therapy targeting apolipoprotein(a): a randomised, double-blind, placebocontrolled phase 1 study. Lancet. 2015;386:1472-83. 
67. Viney NJ, van Capelleveen JC, Geary RS, et al. Antisense oligonucleotides targeting apolipoprotein(a) in people with raised lipoprotein(a): two randomised, double-blind, placebo-controlled, dose-ranging trials. Lancet. 2016;388:2239-53.

68. Ozcan G, Ozpolat B, Coleman RL, Sood AK, Lopez-Berestein G. Preclinical and clinical development of siRNA-based therapeutics. Adv Drug Deliv Rev. 2015;87:108-19.

69. Carthew RW, Sontheimer EJ. Origins and mechanisms of miRNAs and siRNAs. Cell. 2009;136:642-55.

70. Kim DH, Rossi J]. Strategies for silencing human disease using RNA interference. Nat Rev Genet. 2007:8:173-84.

71. Khvorova A. Oligonucleotide therapeutics - A new class of cholesterol-lowering drugs. N Engl J Med. 2017;376:4-7.

72. Juliano R, Alam MR, Dixit V, Kang H. Mechanisms and strategies for effective delivery of antisense and siRNA oligonucleotides. Nucleic Acids Res. 2008;36:4158-71.

73. Fitzgerald $K$, White $S$, Borodovsky $A$, et al. A highly durable RNAi therapeutic inhibitor of PCSK9. N Engl J Med. 2017;376:41-51.
74. Mali $P$, Yang $L$, Esvelt KM, et al. RNA-guided human genome engineering via cas9. Science. 2013;339:823-6.

75. Musunuru K. Genome editing: the recent history and perspective in cardiovascular diseases. J Am Coll Cardiol. 2017;70:2808-21.

76. Cong L, Ran FA, Cox D, et al. Multiplex genome engineering using CRISPR/Cas systems. Science. 2013;339:819-23.

77. Gilbert LA, Larson MH, Morsut L, et al. CRISPR-mediated modular RNA-guided regulation of transcription in eukaryotes. Cell. 2013;154:442-51.

78. Ran FA, Cong L, Yan WX, et al. In vivo genome editing using Staphylococcus aureus cas9. Nature. 2015;520:186-91.

79. Chadwick AC, Wang $X$, Musunuru K. In vivo base editing of PCSK9 (Proprotein convertase subtilisin/Kexin Type 9) as a therapeutic alternative to genome editing. Arterioscler Thromb Vasc Biol. 2017;37:1741-7.

80. Chadwick AC, Evitt NH, Lv W, Musunuru K. Reduced blood lipid levels with in vivo CRISPR-cas9 base editing of ANGPTL3. Circulation. 2018;137:975-7. 